\title{
CORRIGENDUM
}

\section{Ultralow-voltage field-ionization discharge on whiskered silicon nanowires for gas-sensing applications}

Ramin Banan Sadeghian and M. Saif Islam

Nature Materials 10, 135-140 (2011); published online: 16 January 2011; corrected after print: 1 February 2011.

In the version of this Article originally published, the corresponding author and the Acknowledgements were incorrect. These errors have now been corrected in the HTML and PDF versions of the text. 